\title{
Assessment of follicle population changes in sows from day of weaning and during estrus using real-time ultrasound
}

\author{
R. Knox, J. Taibl, M. Altmyer, S. Breen, D. Canaday and A. Visconti \\ Department of Animal Sciences, University of Illinois, Urbana, IL USA
}

Follicle selection and maturation for determining ovulation rate in the pig appears to occur during much of the follicular phase and up to the time of ovulation. There is evidence to suggest that counts of follicles classified as ovulatory-sized at onset of estrus may not reflect the final corpora lutea counts. Follicle size heterogeneity has been reported at estrus and may be related to increased embryonic asynchrony and mortality (Hunter et al. 1989). It is not clear which follicles ovulate at estrus but reports indicate follicles $>4 \mathrm{~mm}$ are LH responsive (Dufour \& Mariana 1993; Lucy et al. 2001). Counts and size measures for ovulatory follicles may differ by the follicle size classification system and in response to whether follicles were assessed by physical or ultrasound measurement (Soede et al. 1998; Knox et al. 2002; Bracken et al. 2006). We hypothesized that the numbers of follicles classified as ovulatory at estrus may not reflect expected ovulation rate or litter size. We performed two experiments to characterize the changes in follicle populations from onset of estrus (Experiment 1) and from time of weaning to ovulation (Experiment 2).

In experiment 1, our objectives were to measure proportions of weaned sows having large, medium and small follicles on the two days before ovulation. A total of 21 sows that had expressed estrus and ovulated between day 2 and 3 and which had real-time ultrasound digital video recordings for both the left and right ovaries on the first (period 1) and second day (period 2) of estrus were included in this study. The images of the ovaries were obtained transrectally using an Aloka $500 \mathrm{~V}$ ultrasound with a $7.5 \mathrm{MHz}$ linear transducer. The images were digitally recorded and follicles individually counted and measured using a digital display system that was calibrated to the measures of the ultrasound. The follicles were classified as small $(\mathrm{S},<3.5$ $\mathrm{mm}$ ), Medium 1 (M1, 3.5-4.99 mm), Medium 2 (M2, 5.0-6.49 mm), Large 1 (L1, 6.5-7.99 mm), Large $2(\mathrm{~L} 2,8.0-9.49 \mathrm{~mm})$, and Large $3(\mathrm{~L} 3,9.5-12.0 \mathrm{~mm})$. Data were analyzed using the GLM procedures of SAS for the main effects of sow and period (day 1 and 2 of estrus). The response measures included the proportions of sows having the specified size class, numbers of follicles in class, and the size of the follicles.

Period did not affect the percentage $(22 \%)$, number $(<3$ follicles), or size of small follicles $(3.1 \mathrm{~mm})$. Period during estrus also did not affect the percentage of sows with $M 1$ follicles $(90 \%)$, or their size $(4.4 \mathrm{~mm})$, but numbers were reduced $(P<0.05)$ in period $2(6.5$ vs. 4.5$)$. Period did not affect the percentage of sows with $(100 \%)$ or numbers ( 14.1 follicles) of $M 2$, but size was increased $(P<0.05)$ in period $2(5.8 \mathrm{vs} .5 .9 \mathrm{~mm})$. Period did not affect the percentage of sows having $\mathrm{L} 1(100 \%)$, but numbers $(7.8$ vs. $9.1, \mathrm{P}=0.01)$ and size $(7.2 \mathrm{vs} .7 .3, \mathrm{P}<0.05)$ were both increased in period 2. Period tended $(P<0.10)$ to increase the percentage of sows having $L 2$ in period 2 compared to period 1 ( $37 \mathrm{vs.} 63 \%$ ) but there was no affect on numbers (2.2) or size of these follicles $(8.6 \mathrm{~mm})$. There was no effect of period on the proportion of sows having $L 3(14 \%)$, or their number $(1.7)$, or their size $(10.8 \mathrm{~mm})$. In assessing the total number of large follicles (all those $>6.49 \mathrm{~mm}$ ), there were more $(P=0.01)$ large follicles in period 
2 (10.6) compared to period 1 (7.9). Since numbers of follicles $>6.49 \mathrm{~mm}$ were increased at period 2, it is evident that growth continues during estrus. Further, since numbers of large follicles were lower than expected ovulation rate for this population of sows $(>16 \mathrm{CL})$, it appears that follicles must ovulate from the larger $M 2$ follicle class to achieve final ovulation rate. The results of this study support the observation for heterogeneity of ovulatory follicles at estrus and could help explain how follicle size heterogeneity might contribute to variation in oocyte and embryo quality and survival.

Experiment 2 was performed using 16 sows that were scanned daily from weaning until ovulation. Data were included only from sows that expressed estrus and ovulated and displayed no ovarian cysts. All sows in this study were $3^{\text {rd }}$ parity PIC C-22 sows, which displayed a weaning to estrus of $5.2 \pm 0.2$ days and ovulation on day $6.1 \pm 0.05$. All sows were mated twice at onset of estrus resulting in an $87 \%$ pregnancy rate, $80 \%$ farrowing rate, 12.9 total born, and 10.8 pigs born alive. Data were analyzed by sow and day of weaning (d 0$)$. Sow had no impact on small or M1 follicles but did impact $M 2$ and larger follicles. Day post weaning had a significant effect on most follicle parameters assessed. The numbers of small follicles differed by day $(P<0.001)$ with more than 12 small follicles on $d 1$ and $<5$ on days $2-6$. Most sows $(>84 \%$ ) maintained small follicles on d $1-2$, but few had small follicles on days 4-6. $\mathrm{M} 1$ follicles were present for most sows ( $>84 \%$ ) on all days but numbers differed by day and ranged from 3-9 follicles. The number of $M 2$ follicles/sow $(n-6)$ did not differ by day but percentages of sows with $M 2$ follicles were $50 \%$ on day 1 and $\geq 85 \%$ on days $2-6$. The number of $L 1$ follicles differed by sow and day. Sows had $\leq 3 L 1$ follicles on $d 1-3$, and on $d 5$ and 6 , increased their numbers to 5-6 L1 follicles. The percentage of sows with $L 1$ follicles was low on $\mathrm{d} 1$, and reaching $46 \%$ on $\mathrm{d} 2$, and then increasing to $>85 \%$ of sows on $\mathrm{d} 3-6$. The L2 class was not present on d $1-2$ but was present in $\sim 50 \%$ of sows on days $3-4$, and in $>80 \%$ of sows on d $5-6$. The $\mathrm{L} 3$ class was represented in only $10 \%$ of sows and estimates were $<1$ follicle on average. The total numbers of follicles $>6.49 \mathrm{~mm}$ on day 1 of estrus was 8.3 with the total number of large follicles reduced on the second day of estrus since ovulation was already in process in half of the sows. Since total numbers of large follicles was lower than CL counts (11.3) in sows that could be scanned for CL counts, and was also lower than total born pigs, we analyzed all follicles $\geq 5.0 \mathrm{~mm}$. The result of the new count was that there were 14.6 follicles at estrus and suggests that ultrasound measures should include follicles $5.0 \mathrm{~mm}$ follicles at onset of estrus for assessment of ovulation rate. In summary, it would appear that for experiments 1 and 2, heterogeneity occurs in follicle sizes that ovulate, and that follicles $5.0 \mathrm{~mm}$ or greater at onset of estrus in weaned sows will ovulate and contribute oocytes that will effect eventual for litter size.

Bracken C J, Radcliff RP, McCormack BL, Keisler DH \& Lucy MC 2006 Decreased follicular size during late lactation caused by treatment with charcoal-treated follicular fluid delays onset of estrus and ovulation after weaning in sows. Journal of Animal Science 84 2110-2117.

Dufour J, \& Mariana JC 1993 Comparative follicular development in Meishan and Large White gilts during prepubertal periods and its relation to hormonal stimulation. Biolog of Reproduction 48 1020-1025.

Hunter MG, Grant SA \& Foxcroft GR 1989 Histological evidence for heterogeneity in the development of preovulatory pig follicles. Journal of Reproduction and Fertility 86 165-170.
Knox RV, Miller GM, Willenburg K.\& Rodriguez-Zas SL. 2002 Effect of frequency of boar exposure and adjusted mating times on measures of reproductive performance in weaned sows. Journal of Animal Science 80 892-899.

Lucy MC, Liu J, Boyd CK \& Bracken CJ 2001 Ovarian follicular growth in sows. Reproduction Supplement $5831-45$.

Soede N, Hazeleger W \& Kemp B 1998 Follicle size and the process of ovulation in sows as studied with ultrasound. Reproduction in Domestic Animals 33 239-244. 